\title{
Practical synthesis of a tetrasaccharide derivative corresponding to ristomycin $\mathrm{A}$ and ristocetin $\mathrm{A}$
}

\author{
Meimei Zhang, Yuguo Du, Fanzuo Kong* \\ Research Center for Eco-Environmental Sciences, Academia Sinica, PO Box 2871, \\ Beijing 100085, People's Republic of China
}

Received 30 August 2000; accepted 16 November 2000

\begin{abstract}
A practical synthesis of fully benzoylated tetrasaccharide, whose free form is indispensable to the antibiotic ristomycin A for the process of dimerization and binding to the cell wall, was achieved via sequential assembly of the building blocks, allyl 3,4-di- $O$-benzoyl- $\alpha$-D-glucopyranoside, 2,3,4-tri- $O$-benzoyl- $\alpha$-L-rhamnopyranosyl trichloroacetimidate, 2- $O$-acetyl-3,4,6-tri- $O$-benzoyl- $\alpha$-D-mannopyranosyl trichloroacetimidate, and 2,3,5-tri- $O$-benzoyl- $\alpha$-D-arabinofuranosyl trichloroacetimidate. A one-pot preparation of allyl 3,4-di-O-benzoyl-2-O-tertbutyldimethylsilyl-6- $O$-triphenylmethyl- $\alpha$-D-glucopyranoside is described, and regioselective glycosylation is carried out using perbenzoylated sugar trichloroacetimidates as glycosyl donors in the presence of a catalytic amount of trimethylsilyl trifluoromethanesulfonate (TMSOTf). (C) 2001 Elsevier Science Ltd. All rights reserved.
\end{abstract}

Keywords: Ristomycin; Ristocetin; Regioselective reaction; Oligosaccharide

\section{Introduction}

The antibiotic, ristomycin $\mathrm{A}$, which is a member of the vancomycin group of antibiotics, is a glycopeptide containing a complex peptide aglycone moiety and a branched heterotetrasaccharide side chain. Ristomycin A, together with its chemical and biological analogue ristocetin $\mathrm{A}$, have emerged as promising antibiotics in the treatment of Gram-positive bacterial infections. ${ }^{1}$ It has been well established that the biological action of vancomycin-type antibiotics involves reversible binding to nascent bacterial cell-wall peptides terminating in L-Lys-D-Ala-D-Ala through hydrogen bonding. ${ }^{1,2}$ This action is

* Corresponding author. Tel.: + 86-10-62936613; fax: + 86-10-62923563.

E-mail address: fzkong@mail.rcees.ac.cn (F. Kong). also slightly adjusted by the formation of homodimers between two antibiotic monomers. ${ }^{1}$ Structural elucidation of ristomycin $\mathrm{A}$ and ristocetin A via acetolysis yielded a fully acetylated tetrasaccharide that was determined to have the following sugar sequence: $\alpha$-D-arabinopyranosyl- $(1 \rightarrow 2)$ - $\alpha$-D - mannopyranosyl$(1 \rightarrow 2)$ - $[\alpha$ - L-rhamnopyranosyl- $(1 \rightarrow 6)]-\beta$ - Dglucopyranose. ${ }^{3,4}$ Later, this structure was revised to $\alpha$-D-arabinofuranosyl- $(1 \rightarrow 2)-\alpha$-Dmannopyranosyl - $(1 \rightarrow 2)$ - $[\alpha$ - L - rhamnopyranosyl-( $(1 \rightarrow 6)]-\beta$-D-glucopyranose by more extensive spectroscopic studies, i.e., the $\alpha$-furanosyl structure for the D-arabinosyl unit was assigned to the oligosaccharide moiety. ${ }^{5-7}$

The tetrasaccharide residue has proved to be indispensable to the biological processing of ristomycin $\mathrm{A}$ and ristocetin $\mathrm{A}$, such as in the process of dimerization and binding to the cell wall. ${ }^{8}$ Herein, we report the synthesis of a ristomycin-related heterotetrasaccharide by a 
simple method in which a one-pot synthesis of the 2,6-differentiated $\alpha$-D-glucopyranoside and a regioselective glycosylation were employed.

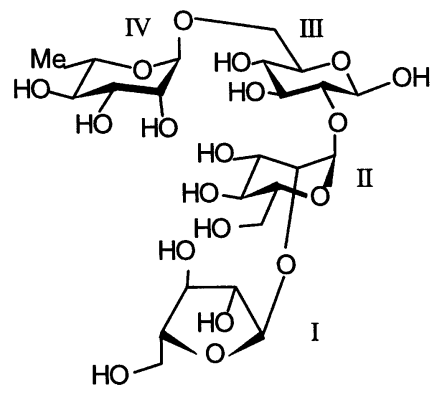

\section{Results and discussion}

We have previously shown ${ }^{9}$ that the use of benzoylated imidates as glycosyl donors gave good yields and regioselectivities in glycosylations. Besides, the common acyl protection group used in the synthesis greatly simplified both the preparation of building blocks and the final deprotection of the product. Accordingly, we designed a practical strategy for the synthesis of the ristomycin A (or ristocetin A) related sugar residue using the building blocks 4, 7, 10 and 13.

Synthon 4 was synthesized via a one-pot, three-step sequential reaction ${ }^{10}$ as follows. Allyl $\alpha$-D-glucopyranoside (1) was treated with 1.25 equiv of chlorotriphenylmethane $(\mathrm{Tr})$ and a catalytic amount of 4-dimethylaminopyridine (DMAP) in pyridine at $80^{\circ} \mathrm{C}$. Without working up, the mixture was regioselectively silylated with 1.1 equiv of tert-butylchlorodimethylsilane (TBDMSCl) and 2 equiv of imidazole, then benzoylated with benzoyl chloride to give allyl 3,4-di- $O$-benzoyl-2- $O$ tert-butyldimethylsilyl-6- $O$-triphenylmethyl- $\alpha$ D-glucopyranoside (2) in a total yield of $79 \%$. The ${ }^{1} \mathrm{H}$ NMR spectrum of 2 gave $\mathrm{H}-2$ (4.0 ppm) and H-6 (3.21, $3.25 \mathrm{ppm})$ as upfield doublets of doublets, while $\mathrm{H}-3$ (5.80 ppm) and $\mathrm{H}-4$ (5.32 ppm) appeared as downfield triplets, which clearly indicates the correct structure of $\mathbf{2}$ (see Scheme 1).

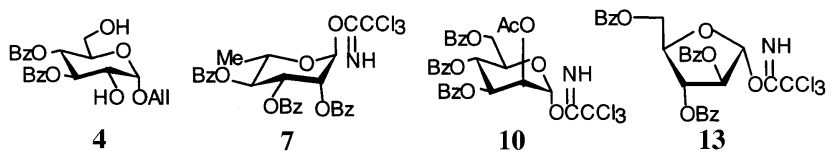

Treatment of 2 with $90 \%$ trifluoroacetic acid (TFA) at room temperature gave diol $\mathbf{4}$ in $91 \%$ yield. Attempted detritylation of $\mathbf{2}$ with $>2$ equiv of ferric chloride hexahydrate in $\mathrm{CH}_{2} \mathrm{Cl}_{2}{ }^{11}$ was not satisfactory, and monosilylated compounds 3 and diol $\mathbf{4}$ were obtained in a ratio of $1: 1$. Elongation of the reaction time generated 4 as a final product, while adding 1.5 equiv of $\mathrm{FeCl}_{3} \cdot 6 \mathrm{H}_{2} \mathrm{O}$ gave 3 and 4 in a $4: 1$ ratio for $1.5 \mathrm{~h}$ at $0{ }^{\circ} \mathrm{C}$.

Regioselective debenzoylation of fully benzoylated L-rhamnopyranose (5) on $\mathrm{C}$-1 with $\mathrm{NH}_{3}$ in 3:7 $\mathrm{CH}_{3} \mathrm{OH}-\mathrm{THF}(\rightarrow \mathbf{6})$, followed by anomeric activation with $\mathrm{CCl}_{3} \mathrm{CN}$ and 1,8-diazabicyclo[5.4.0]undec-7-ene (DBU) gave the

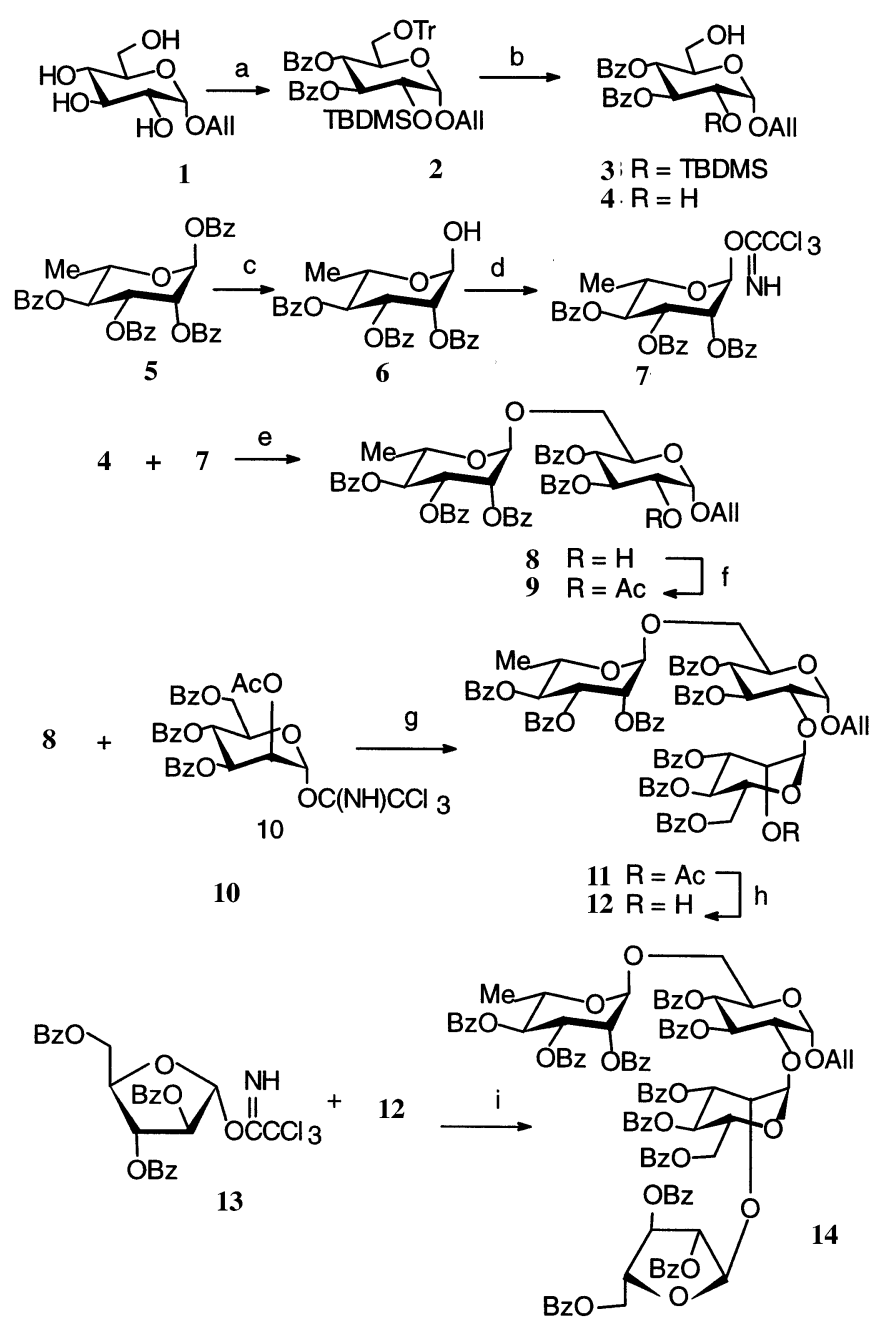

Scheme 1. (a) TrCl, Py, DMAP; Im, TBDMSCl in DMF; $\mathrm{BzCl}$, Py; (b) $\mathrm{FeCl}_{3} \cdot 6 \mathrm{H}_{2} \mathrm{O}, \mathrm{DCM}$; (c) $\mathrm{NH}_{3}, 3: 7 \mathrm{CH}_{3} \mathrm{OH}-$ THF; (d) DBU, $\mathrm{CCl}_{3} \mathrm{CN}$; (e) TMSOTf, $<-15^{\circ} \mathrm{C}_{2} \mathrm{CH}_{2} \mathrm{Cl}_{2}$ (anhyd); (f) $\mathrm{Ac}_{2} \mathrm{O}$, Py; (g) TMSOTf, $0{ }^{\circ} \mathrm{C}, \mathrm{CH}_{2} \mathrm{Cl}_{2}$ (anhyd); (h) $3 \% \mathrm{CH}_{3} \mathrm{COCl}, 1: 1 \mathrm{CH}_{3} \mathrm{OH}-\mathrm{CH}_{2} \mathrm{Cl}_{2}$; (i) TMSOTf, $0{ }^{\circ} \mathrm{C}$, $\mathrm{CH}_{2} \mathrm{Cl}_{2}$ (anhyd). 
2,3,4 - tri - $O$ - benzoyl - $\alpha$ - L - rhamnopyranosyltrichloroacetimidate (7) in a total yield of $76 \%$. Regioselective coupling of 7 with diol 4 in anhydrous $\mathrm{CH}_{2} \mathrm{Cl}_{2}$ in the presence of $\mathrm{TM}$ SOTf ( 0.1 equiv) at $-15^{\circ} \mathrm{C}$ furnished $1 \rightarrow 6$ linked disaccharide $\mathbf{8}$ in $72.5 \%$ yield after column chromatography. To prove the coupling reaction actually occurred at C-6 of acceptor 4, disaccharide 8 (20 $\mathrm{mg})$ was treated with $\mathrm{Ac}_{2} \mathrm{O}$ in pyridine to give the $\mathrm{C}-2$ acetylated derivative 9. The ${ }^{1} \mathrm{H}$ NMR spectrum of 9 clearly showed an downfield shift of $\mathrm{H}-2$ (from 3.90 to $5.17 \mathrm{ppm}$ ), indicating the presence of a free $\mathrm{C}-2$ hydroxyl group in $\mathbf{8}$. The TMSOTf ( 0.15 equiv) promoted coupling reaction of 2-O-acetyl-3,4,6-tri- $O$-benzoyl$\alpha$-D-mannopyranosyl trichloroacetimidate (10) with 8 at $0{ }^{\circ} \mathrm{C}$ afforded trisacchride 11 in high yield $(83 \%)$. The structure of 11 was fully assigned from its ${ }^{1} \mathrm{H}-{ }^{1} \mathrm{H}$ COSY NMR spectroscopy. The stereochemistry with all $\alpha$ glycosidic linkages in $\mathbf{1 1}$ was established by measuring the ${ }^{1} J_{\mathrm{C}-1, \mathrm{H}-1}$ heteronuclear coupling constants $\left({ }^{1} J_{\mathrm{C}-1, \mathrm{H}-1}\right.$ with 173,172 and 168 $\mathrm{Hz}$ at $\delta 98.4,94.8$ and 93.7 ppm, respectively). No orthoester intermediate was detected in this case. Compound $\mathbf{1 1}$ was selectively deacetylated $^{12}$ with acetyl chloride $(3 \%$ in $1: 1$ $\mathrm{CH}_{3} \mathrm{OH}-\mathrm{CH}_{2} \mathrm{Cl}_{2}$ ) at room temperature for $20 \mathrm{~h}$ to get exclusively the corresponding trisaccharide acceptor 12. The chemical shift of $\mathrm{H}-2$ on the mannose residue was moved upfield from 5.45 (in 11) to $4.41 \mathrm{ppm}$ (in 12), strongly supporting the fact that no acyl migration occurred under these reaction conditions. Similarly, the TMSOTf $(0.1$ equiv $)$ promoted the coupling reaction of 2,3,5-tri- $O$ benzoyl- $\alpha$-D-arabinofuranosyl 2,2,2-trichloroacetimidate $(\mathbf{1 3})^{13}$ with $\mathbf{1 2}$ in anhydrous dichloromethane at $0{ }^{\circ} \mathrm{C}$ afforded the target tetrasaccharide $\mathbf{1 4}$ in $80 \%$ yield. This tetrasaccharide could be useful in the total synthesis of ristomycin $\mathrm{A}$ or ristocetin $\mathrm{A}^{14}$ after transformation into a glycosyl donor via deallylation and activation. ${ }^{9}$ Furthermore, we proved here a practical one-pot preparation of 2,6-differentiated $\alpha$-D-glucopyranoside that may provide an effective strategy for the synthesis of 2,6-branched oligosaccharides.

\section{Experimental}

General methods.-Optical rotations were determined at $25^{\circ} \mathrm{C}$ with a Perkin-Elmer model 241MC automatic polarimeter. ${ }^{1} \mathrm{H}$ NMR and ${ }^{1} \mathrm{H}-{ }^{1} \mathrm{H}$ COSY spectra were recorded with a Bruker ARX 400 spectrometer for solutions in $\mathrm{CDCl}_{3}$. Chemical shifts are given in ppm downfield from internal $\mathrm{Me}_{4} \mathrm{Si}$. Mass spectra were recorded with a VG PlARFOEM mass spectrometer using the FAB technique to introduce the sample. Thin-layer chromatography (TLC) was performed on Silica Gel $\mathrm{HF}_{254}$ (E. Merck) with detection by charring with $30 \%(\mathrm{v} / \mathrm{v}) \mathrm{H}_{2} \mathrm{SO}_{4}$ in $\mathrm{MeOH}$, or in some cases by a UV detector. Column chromatography was conducted by elution of a column of silica gel (100-200 mesh) with EtOAc-petroleum ether (bp $60-90{ }^{\circ} \mathrm{C}$ ) or in some cases with toluene-EtOAc-petroleum ether as the eluent. Solutions were concentrated at $<80{ }^{\circ} \mathrm{C}$ under diminished pressure.

Allyl 3,4-di-O-benzoyl-2-O-tert-butyldimethylsilyl-6-O-triphenylmethyl- $\alpha$-D-glucopyranoside (2)._Compound $\mathbf{1}^{15}$ (5 g, $\left.22.7 \mathrm{mmol}\right)$ was dissolved in pyridine $(25 \mathrm{~mL})$. A catalytic amount of DMAP was added into the solution. Chlorotriphenylmethane (7.6 g, 27.3 mmol) was then added. The mixture was stirred at $80{ }^{\circ} \mathrm{C}$ for $16 \mathrm{~h}$, then cooled down to $0{ }^{\circ} \mathrm{C}$. Imidazole $(3.10 \mathrm{~g}, 45 \mathrm{mmol})$ was added; then TBDMSCl $(3.75 \mathrm{~g}, 25 \mathrm{mmol})$ in DMF (5 $\mathrm{mL})$ was added portionwise over $2 \mathrm{~h}$. The mixture was stirred at $\mathrm{rt}$ for $6 \mathrm{~h}$, at which time premixed $\mathrm{BzCl}(7 \mathrm{~mL}, 55 \mathrm{mmol})$ and pyridine $(5 \mathrm{~mL})$ was added. The reaction mixture was stirred at $50{ }^{\circ} \mathrm{C}$ for $24 \mathrm{~h}$, then co-evaporated with toluene to dryness. The residue was dissolved in $\mathrm{CH}_{2} \mathrm{Cl}_{2}(150 \mathrm{~mL})$ and washed with ice-cold water. The washings were re-extracted with $\mathrm{CH}_{2} \mathrm{Cl}_{2}$. The combined organic phase was dried, concentrated and subjected to column chromatography on silica gel with 15:1 petroleum ether-EtOAc as the eluent to give $2(14 \mathrm{~g}, 79 \%)$ as a syrup; $[\alpha]_{\mathrm{D}}^{25}+1^{\circ}(c 4.1$, $\left.\mathrm{CHCl}_{3}\right) ;{ }^{1} \mathrm{H} \mathrm{NMR}\left(\mathrm{CDCl}_{3}\right): \delta 0.05,0.14(2 \mathrm{~s}$, $\left.2 \times 3 \mathrm{H}, \mathrm{Si}\left(\mathrm{CH}_{3}\right)_{2}\right), 0.77$ (s, $\left.9 \mathrm{H}, t-\mathrm{Bu}\right), 3.19-$ 3.27 (m, 2 H, H-6a, H-6b), 4.00 (dd, $1 \mathrm{H}, J_{1,2}$ 3.6, $\left.J_{2,3} 9.6 \mathrm{~Hz}, \mathrm{H}-2\right), 4.14-4.19$ (m, $2 \mathrm{H}, \mathrm{H}-5$, $\left.\mathrm{CH}_{2}=\mathrm{CH}-\mathrm{CH}_{2}-\right), 4.34(\mathrm{dd}, 1 \mathrm{H}, J$ 5.6, 12.8 $\left.\mathrm{Hz}, \mathrm{CH}_{2}=\mathrm{CH}-\mathrm{CH}_{2}-\right), 4.97\left(\mathrm{~d}, 1 \mathrm{H}, J_{1,2} 4.0 \mathrm{~Hz}\right.$, 
$\mathrm{H}-1), \quad 5.26$ (dd $1 \mathrm{H}, \quad J \quad 10.4,1.6 \mathrm{~Hz}$, $\left.\mathrm{CH}_{2}=\mathrm{CH}-\mathrm{CH}_{2}-\right), 5.32\left(\mathrm{t}, 1 \mathrm{H}, J_{3,4} 10 \mathrm{~Hz}\right.$, $\mathrm{H}-4), 5.42$ (dd, $1 \mathrm{H}, J \mathrm{l}, 6,10.4 \mathrm{~Hz}$, $\left.\mathrm{CH}_{2}=\mathrm{CH}-\mathrm{CH}_{2}-\right), 5.80\left(\mathrm{t}, 1 \mathrm{H}, J_{3,4} 9.6 \mathrm{~Hz}\right.$, $\mathrm{H}-3), 6.01-6.03$ (m, $1 \mathrm{H}, \mathrm{CH}_{2}=\mathrm{CH}-\mathrm{CH}_{2}-$ ), 7.01-7.91 (m, 25 H, 5 Ph). Anal. Calcd for $\mathrm{C}_{48} \mathrm{H}_{52} \mathrm{O}_{8} \mathrm{Si}: \mathrm{C}, 73.47 ; \mathrm{H}$, 6.63. Found: C, 73.69; H, 6.56.

Allyl 3,4-di-O-benzoyl-2-O-tert-butyldimethylsilyl- $\alpha$-D-glucopyranoside (3) and 3,4-di-Obenzoyl- $\alpha$-D-glucopyranoside (4). - Method A: $\mathrm{FeCl}_{3} \cdot 6 \mathrm{H}_{2} \mathrm{O}$ (2.5 equiv) was added to a mixture of 2 ( $3 \mathrm{~g}, 3.8 \mathrm{mmol})$ in $\mathrm{CH}_{2} \mathrm{Cl}_{2}(20 \mathrm{~mL})$. The mixture was stirred for $2 \mathrm{~h}$ at $\mathrm{rt}$, then diluted with more $\mathrm{CH}_{2} \mathrm{Cl}_{2}(50 \mathrm{~mL})$ and washed with ice-cold water twice. The washings were re-extracted with $\mathrm{CH}_{2} \mathrm{Cl}_{2}(30 \mathrm{~mL})$. The organic phases were combined, dried and concentrated, then subjected to column chromatography on silica gel with 4:1-1.5:1 petroleum ether-EtOAc as the eluent to give syrupy 3 (1.06 g, 51\%) and syrupy 4 (800 mg, 49\%), respectively. Method B: Compound 2 $(2.5 \mathrm{~g}, 3.2 \mathrm{mmol})$ was dissolved into $90 \% \mathrm{aq}$ TFA $(15 \mathrm{~mL})$. The mixture was stirred at $\mathrm{rt}$ for $2 \mathrm{~h}$, then toluene $(50 \mathrm{~mL})$ was added, and the solvents were evaporated in vacuo to give a residue, which was purified by a silica gel column chromatography $(1: 1$ petroleum ether-EtOAc) to give $4(1.26 \mathrm{~g}, 92 \%)$ as a syrup. Compound 3: $[\alpha]_{\mathrm{D}}^{25}-12^{\circ}$ (c) 1.3 , $\left.\mathrm{CHCl}_{3}\right) ;{ }^{1} \mathrm{H}$ NMR $\left(\mathrm{CDCl}_{3}\right): \delta 0.04(2 \mathrm{~s}, 2 \times 3$ $\left.\mathrm{H}, \mathrm{Si}\left(\mathrm{CH}_{3}\right)_{2}\right), 0.77$ (s, $\left.9 \mathrm{H}, \mathrm{t}-\mathrm{Bu}\right), 3.66(\mathrm{dd}, 1$ $\left.\mathrm{H}, J_{5,6 \mathrm{a}} 2.8, J_{6 \mathrm{a}, 6 \mathrm{~b}} 13.2 \mathrm{~Hz}, \mathrm{H}-6 \mathrm{a}\right), 3.76(\mathrm{dd}, 1$ $\left.\mathrm{H}, J_{5,6 \mathrm{~b}} 2.0, J_{6 \mathrm{a}, 6 \mathrm{~b}} 12.8 \mathrm{~Hz}, \mathrm{H}-6 \mathrm{~b}\right), 3.97-3.99$ $(\mathrm{m}, 1 \mathrm{H}, \mathrm{H}-5), 4.00\left(\mathrm{dd}, 1 \mathrm{H}, J_{1,2} 3.6, J_{2,3} 9.6\right.$ $\mathrm{Hz}, \mathrm{H}-2), 4.10$ (dd, $1 \mathrm{H}, J 5.6,13.0 \mathrm{~Hz}$, $\left.\mathrm{CH}_{2}=\mathrm{CH}-\mathrm{CH}_{2}-\right), 4.29(\mathrm{dd}, 1 \mathrm{H}, J$ 5.6, 13.0 $\left.\mathrm{Hz}, \mathrm{CH}_{2}=\mathrm{CH}-\mathrm{CH}_{2}-\right), 4.96\left(\mathrm{~d}, J_{1,2} 4.0 \mathrm{~Hz}\right.$, $\mathrm{H}-1), 5.25$ (d, J $10.4 \mathrm{~Hz}, \mathrm{CH}_{2}=\mathrm{CH}-\mathrm{CH}_{2}-$ ), $5.34\left(\mathrm{t}, J_{3,4} 10 \mathrm{~Hz}, \mathrm{H}-4\right), 5.39$ (dd, $1 \mathrm{H}, J 1.6$, $\left.10.4 \mathrm{~Hz}, \mathrm{CH}_{2}=\mathrm{CH}-\mathrm{CH}_{2}-\right), 5.96\left(\mathrm{t}, J_{3,4} 10 \mathrm{~Hz}\right.$, $\mathrm{H}-3)$, 5.92-6.02 (m, $1 \mathrm{H}, \mathrm{CH}_{2}=\mathrm{CH}-\mathrm{CH}_{2}-$ ), 7.26-8.10 (m, 10 H, 2 Ph). Compound 4: $[\alpha]_{\mathrm{D}}^{25}$ $+26^{\circ}\left(\right.$ c 2.1, $\left.\mathrm{CHCl}_{3}\right)$; ${ }^{1} \mathrm{H}$ NMR $\left(\mathrm{CDCl}_{3}\right): \delta$ $3.68\left(\mathrm{dd}, 1 \mathrm{H}, J_{5,6 \mathrm{a}} 3.6, J_{6 \mathrm{a}, 6 \mathrm{~b}} 13 \mathrm{~Hz}, \mathrm{H}-6 \mathrm{a}\right)$, 3.77 (dd, $\left.1 \mathrm{H}, J_{5,6 \mathrm{~b}} 2.4, J_{6 \mathrm{a}, 6 \mathrm{~b}} 13 \mathrm{~Hz}, \mathrm{H}-6 \mathrm{~b}\right)$, $3,91\left(\mathrm{dd}, 1 \mathrm{H}, J_{1,2} 3.6, J_{2,3} 9.6 \mathrm{~Hz}, \mathrm{H}-2\right)$, 3.96-3.98 (m, $1 \mathrm{H}, \mathrm{H}-5), 4.12$ (dd, $1 \mathrm{H}, J$ 5.6, $\left.13.2 \mathrm{~Hz}, \mathrm{CH}_{2}=\mathrm{CH}-\mathrm{CH}_{2}-\right), 4.31(\mathrm{dd}, 1 \mathrm{H}, J$ 5.6, $\left.12.8 \mathrm{~Hz}, \mathrm{CH}_{2}=\mathrm{CH}-\mathrm{CH}_{2}-\right), 5.10(\mathrm{~d}, 1 \mathrm{H}$,
$\left.J_{1,2} 4.0 \mathrm{~Hz}, \mathrm{H}-1\right), 5.28(\mathrm{~d}, 1 \mathrm{H}, J 10.4 \mathrm{~Hz}$, $\left.\mathrm{CH}_{2}=\mathrm{CH}-\mathrm{CH}_{2}-\right), 5.38\left(\mathrm{t}, 1 \mathrm{H}, J_{3,4} 10 \mathrm{~Hz}\right.$, $\mathrm{H}-4), 5.40\left(\mathrm{~m}, 1 \mathrm{H}, \mathrm{CH}_{2}=\mathrm{CH}-\mathrm{CH}_{2}-\right), 5.80(\mathrm{t}, 1$ $\left.\mathrm{H}, J_{3,4}=J_{2,3}=9.6 \mathrm{~Hz}, \mathrm{H}-3\right), 5.92-6.02(\mathrm{~m}, 1$ $\left.\mathrm{H}, \mathrm{CH}_{2}=\mathrm{CH}-\mathrm{CH}_{2}-\right), 7.33-7.97(\mathrm{~m}, 10 \mathrm{H}, 2$ $\mathrm{Ph}$ ). Anal. Calcd for $\mathrm{C}_{23} \mathrm{H}_{24} \mathrm{O}_{8}$ : C, 64.48; $\mathrm{H}$, 5.61. Found: C, 64.21; H, 5.54.

2,3,4-Tri-O - benzoyl- $\alpha$ - L - rhamnopyranosyl trichloroacetimidate (7).-Ammonium was bubbled into a solution of $5^{16}(6 \mathrm{~g}, 10.3 \mathrm{mmol})$ in 3:7 MeOH-THF $(100 \mathrm{~mL})$ for $15 \mathrm{~min}$. The reaction mixture was stirred at $\mathrm{rt}$ for $3 \mathrm{~h}$, then evaporated to dryness. Purification on a flash silica gel column using 1:2 EtOAc-petroleum ether as eluent gave $\mathbf{6}$, which was dissolved in anhyd $\mathrm{CH}_{2} \mathrm{Cl}_{2}(100 \mathrm{~mL})$. To this solution was added trichloroacetonitrile $(10 \mathrm{~mL}, 100 \mathrm{mmol})$ and DBU $(1.2 \mathrm{~mL})$. The reaction mixture was stirred at $\mathrm{rt}$ for $6 \mathrm{~h}$, then concentrated. The residue was purified by silica gel column chromatography (3:1 petroleum ether-EtOAc) to give $7(4.87 \mathrm{~g}, 76 \%)$ as a syrup; $[\alpha]_{\mathrm{D}}^{25}+110^{\circ}(c$ 6.0, $\left.\mathrm{CHCl}_{3}\right) ;{ }^{1} \mathrm{H} \mathrm{NMR}\left(\mathrm{CDCl}_{3}\right): \delta 1.43(\mathrm{~d}, 3$ $\left.\mathrm{H}, J 6.2 \mathrm{~Hz}, \mathrm{CH}_{3}\right), 4.40-4.44$ (m, $\left.1 \mathrm{H}, \mathrm{H}-5\right)$, $5.78\left(\mathrm{t}, 1 \mathrm{H}, J_{3,4} 10.0 \mathrm{~Hz}, \mathrm{H}-4\right), 5.90-5.93(\mathrm{~m}$, $2 \mathrm{H}, \mathrm{H}-2, \mathrm{H}-3), 6.50$ (d, $\left.1 \mathrm{H}, J_{1,2} 1.6 \mathrm{~Hz}, \mathrm{H}-1\right)$, 7.24-8.12 (m, $15 \mathrm{H}, 3 \mathrm{Ph}), 8.84$ (s, $1 \mathrm{H}, \mathrm{NH})$.

Allyl 2,3,4-tri-O-benzoyl- $\alpha$-L-rhamnopyranosyl- $(1 \rightarrow 6)$ - 3,4-di-O - benzoyl - $\alpha$ - D - glucopyranoside (8). - To a mixture of $4(800 \mathrm{mg}, 1.87$ $\mathrm{mmol})$ and 7 (1.31 g, $2.1 \mathrm{mmol})$ in anhyd $\mathrm{CH}_{2} \mathrm{Cl}_{2}(20 \mathrm{~mL})$ was added $\mathrm{Me}_{3}$ SiOTf $(10 \%$ equiv) dropwise under a $\mathrm{N}_{2}$ atmosphere at $-15^{\circ} \mathrm{C}$. The mixture was stirred under these conditions for $1 \mathrm{~h}$, at the end of which time, TLC (2:1 petroleum ether-EtOAc) indicated that all the starting material had been consumed. The reaction mixture was neutralized with $\mathrm{Et}_{3} \mathrm{~N}$, then concentrated. The product was purified by column chromatography twice (3:1 petroleum ether-EtOAc and 0.5:2.5:1 toluene-petroleum ether-EtOAc) to give $\mathbf{8}$ (1.2 $\mathrm{g}, \quad 72.5 \%) ;[\alpha]_{\mathrm{D}}^{25}+84^{\circ}\left(c \quad 0.9, \mathrm{CHCl}_{3}\right) ;{ }^{1} \mathrm{H}$ NMR $\left(\mathrm{CDCl}_{3}\right): \delta 1.26(\mathrm{~d}, 3 \mathrm{H}, J 6.4 \mathrm{~Hz}$, $\left.\mathrm{CH}_{3}^{\mathrm{IV}}\right), 3.77\left(\mathrm{dd}, 1 \mathrm{H}, J_{5,6 \mathrm{a}} 6.8, J_{6 \mathrm{a}, 6 \mathrm{~b}} 11.6 \mathrm{~Hz}\right.$, $\left.\mathrm{H}-6 \mathrm{a}^{\mathrm{III}}\right), 3.89-3.92\left(\mathrm{~m}, 2 \mathrm{H}, \mathrm{H}-2^{\mathrm{III}}, \mathrm{H}-6 \mathrm{~b}^{\mathrm{III}}\right)$, 4.11-4.12 (m, $\left.1 \mathrm{H}, \mathrm{H}-5^{\mathrm{IV}}\right), 4.25$ (q, $1 \mathrm{H}, J$ 6.0, $\left.12.8 \mathrm{~Hz}, \mathrm{CH}_{2}=\mathrm{CH}-\mathrm{CH}_{2}-\right), 4.29-4.33(\mathrm{~m}, 1 \mathrm{H}$, $\left.\mathrm{H}-5^{\mathrm{III}}\right), 4.45-4.49\left(\mathrm{~m}, 1 \mathrm{H}, \mathrm{CH}_{2}=\mathrm{CH}-\mathrm{CH}_{2}-\right)$, 5.11-5.13 (m, $\left.2 \mathrm{H}, \mathrm{H}-1^{\mathrm{III}}, \mathrm{H}-1^{\mathrm{IV}}\right), 5.33-5.36$ 
$\left(\mathrm{m}, 1 \mathrm{H}, \mathrm{CH}_{2}=\mathrm{CH}-\mathrm{CH}_{2}-\right), 5.42\left(\mathrm{t}, 1 \mathrm{H}, J_{3,4}\right.$ $\left.10.0 \mathrm{~Hz}, \mathrm{H}-4^{\mathrm{III}}\right), \quad 5.47-5.52 \quad(\mathrm{~m}, 1 \mathrm{H}$, $\left.\mathrm{CH}_{2}=\mathrm{CH}-\mathrm{CH}_{2}-\right), 5.63\left(\mathrm{t}, 1 \mathrm{H}, J_{3,4} 10.0 \mathrm{~Hz}\right.$, $\left.\mathrm{H}-3^{\mathrm{III}}\right), 5.71-5.72\left(\mathrm{~m}, 1 \mathrm{H}, \mathrm{H}-2^{\mathrm{IV}}\right), 5.76-5.79$ $\left(\mathrm{m}, 2 \mathrm{H}, \mathrm{H}-3^{\mathrm{IV}}, \mathrm{H}-4^{\mathrm{IV}}\right), 6.06(\mathrm{~m}, 1 \mathrm{H}$, $\left.\mathrm{CH}_{2}=\mathrm{CH}-\mathrm{CH}_{2}-\right)$, 7.25-8.10 (m, $\left.25 \mathrm{H}, 5 \mathrm{Ph}\right)$. Anal. Calcd for $\mathrm{C}_{50} \mathrm{H}_{46} \mathrm{O}_{15}$ : C, 67.72; H, 5.19. Found: C, 67.95; H, 5.33.

Allyl 2,3,4-O-tri-benzoyl- $\alpha$-L-rhamnopyranosyl- $(1 \rightarrow 6)-2-\mathrm{O}$ - acetyl-3,4-di-O-benzoyl- $\alpha$ D-glucopyranoside (9). - Compound 8 (20 mg) was dissolved in pyridine $(1 \mathrm{~mL})$. Then $\mathrm{Ac}_{2} \mathrm{O}$ $(0.5 \mathrm{~mL})$ was added into the solution. The mixture was stirred at rt overnight. The solvents were evaporated under reduced pressure to give 9 for NMR testing; ${ }^{1} \mathrm{H} \mathrm{NMR}\left(\mathrm{CDCl}_{3}\right)$ : $\delta 1.26\left(\mathrm{~d}, 3 \mathrm{H}, J 6.2 \mathrm{~Hz}, \mathrm{CH}_{3}^{\mathrm{IV}}\right), 3.79(\mathrm{dd}, 1 \mathrm{H}$, $\left.J_{5,6 \mathrm{a}} 7.2, J_{6 \mathrm{a}, 6 \mathrm{~b}} 12 \mathrm{~Hz}, \mathrm{H}-6 \mathrm{a}^{\mathrm{III}}\right), 3.89(\mathrm{dd}, 1 \mathrm{H}$, $\left.J_{5,6 \mathrm{~b}} 2.0, J_{6 \mathrm{a}, 6 \mathrm{~b}} 12 \mathrm{~Hz}, \mathrm{H}-6 \mathrm{~b}^{\mathrm{III}}\right), 4.11-4.13(\mathrm{~m}, 1$ $\left.\mathrm{H}, \mathrm{H}-5^{\mathrm{IV}}\right), 4.22(\mathrm{dd}, 1 \mathrm{H}, J 6.0,12.8 \mathrm{~Hz}$, $\left.\mathrm{CH}_{2}=\mathrm{CH}-\mathrm{CH}_{2}-\right), 4.36-4.45\left(\mathrm{~m}, 2 \mathrm{H}, \mathrm{H}-5^{\mathrm{III}}\right.$, $\left.\mathrm{CH}_{2}=\mathrm{CH}-\mathrm{CH}_{2}-\right), 5.11\left(\mathrm{~d}, 1 \mathrm{H}, J_{1^{\prime}, 2^{\prime}} 1.4 \mathrm{~Hz}\right.$, $\left.\mathrm{H}-1^{\mathrm{IV}}\right), 5.17\left(\mathrm{dd}, 1 \mathrm{H}, J_{1,2} 3.6, J_{2,3} 10.4 \mathrm{~Hz}\right.$, H-2 $\left.{ }^{\mathrm{III}}\right)$, 5.24-5.25 (d, $\left.1 \mathrm{H}, J_{1,2} 3.6 \mathrm{~Hz}, \mathrm{H}-1^{\mathrm{III}}\right)$, 5.30-5.32 (m, $\left.1 \mathrm{H}, \mathrm{CH}_{2}=\mathrm{CH}-\mathrm{CH}_{2}-\right), 5.39-$ $5.44\left(\mathrm{t}, 1 \mathrm{H}, J_{3,4} 10.0 \mathrm{~Hz}, \mathrm{H}-4^{\mathrm{III}}\right), 5.46-5.51$ $\left(\mathrm{m}, 1 \mathrm{H}, \mathrm{CH}_{2}=\mathrm{CH}-\mathrm{CH}_{2}-\right), 5.65\left(\mathrm{t}, 1 \mathrm{H}, J_{3^{\prime}, 4^{\prime}}\right.$ $\left.10.0 \mathrm{~Hz}, \mathrm{H}-4^{\mathrm{IV}}\right), 5.69-5.70\left(\mathrm{~m}, 1 \mathrm{H}, \mathrm{H}-2^{\mathrm{IV}}\right)$, $5.77\left(\mathrm{dd}, 1 \mathrm{H}, J_{2^{\prime}, 3^{\prime}} 3.6, J_{3^{\prime}, 4^{\prime \prime}} 10.0 \mathrm{~Hz}, \mathrm{H}-3^{\mathrm{IV}}\right)$, 6.01-6.06 (m, 2 H, H-3, $\mathrm{CH}_{2}=\mathrm{CH}-\mathrm{CH}_{2}-$ ), 7.24-8.09 (m, $25 \mathrm{H}, 5 \mathrm{Ph})$. Anal. Calcd for $\mathrm{C}_{52} \mathrm{H}_{48} \mathrm{O}_{16}: \mathrm{C}, 67.24 ; \mathrm{H}, 5.17$. Found: C, 67.45; $\mathrm{H}, 5.30$.

Allyl 2-O-acetyl-3,4,6-tri-O-benzoyl- $\alpha$-Dmannopyranosyl- $(1 \rightarrow 2)$-[2,3,4-tri-O-benzoyl$\alpha$-L-rhamnopyranosyl- $(1 \rightarrow 6)]-3,4-d i-\mathrm{O}$-benzoyl- $\alpha$-D-glucopyranoside (11). - To a mixture of $8(920 \mathrm{mg}, 1.04 \mathrm{mmol})$ and $\mathbf{1 0}^{17}(815 \mathrm{mg}$, $1.2 \mathrm{mmol})$ in anhyd $\mathrm{CH}_{2} \mathrm{Cl}_{2}(10 \mathrm{~mL})$ was dropped $\mathrm{Me}_{3} \mathrm{SiOTf}\left(10 \%\right.$ equiv) under a $\mathrm{N}_{2}$ atmosphere at $0{ }^{\circ} \mathrm{C}$. The mixture was stirred under these conditions for $2 \mathrm{~h}$, at the end of which time, TLC $(2: 1$ petroleum etherEtOAc) indicated that all the donor was consumed. The reaction mixture was neutralized with $\mathrm{Et}_{3} \mathrm{~N}$, then concentrated. The residue was purified by column chromatography (0.5:2:1 toluene-petroleum ether-EtOAc) to give trisaccharide $11(1.21 \mathrm{~g}, 83 \%)$ as a syrup; $[\alpha]_{\mathrm{D}}^{25}$ $+90^{\circ}\left(\right.$ c 1.2, $\left.\mathrm{CHCl}_{3}\right) ;{ }^{1} \mathrm{H}$ NMR $\left(\mathrm{CDCl}_{3}\right): \delta$ $1.27\left(\mathrm{~d}, 3 \mathrm{H}, J 6.4 \mathrm{~Hz}, \mathrm{CH}_{3}\right), 2.11(\mathrm{~s}, 3 \mathrm{H}$,
$\left.\mathrm{CH}_{3} \mathrm{CO}\right), 3.75\left(\mathrm{dd}, 1 \mathrm{H}, J_{5,6 \mathrm{a}} 7.2, J_{6 \mathrm{a}, 6 \mathrm{~b}} 12.0\right.$ $\left.\mathrm{Hz}, \mathrm{H}-6 \mathrm{a}^{\mathrm{III}}\right), 3.91\left(\mathrm{dd}, 1 \mathrm{H}, J_{5,6 \mathrm{~b}} 2.0, J_{6 \mathrm{a}, 6 \mathrm{~b}} 12.0\right.$ $\left.\mathrm{Hz}, \mathrm{H}-6 \mathrm{~b}^{\mathrm{III}}\right), 4.13-4.33$ (m, $6 \mathrm{H}, \mathrm{H}-2^{\mathrm{III}}, \mathrm{H}-5^{\mathrm{IV}}$, $\left.\mathrm{H}-5^{\mathrm{II}}, \mathrm{H}-6 \mathrm{a}^{\mathrm{II}}, \mathrm{H}-6 \mathrm{~b}^{\mathrm{II}}, \mathrm{CH}_{2}=\mathrm{CH}-\mathrm{CH}_{2}-\right), 4.38-$ $4.42\left(\mathrm{~m}, 1 \mathrm{H}, \mathrm{H}-5^{\mathrm{III}}\right), 4.50(\mathrm{dd}, 1 \mathrm{H}, J 5.2 \mathrm{~Hz}$, $\left.\mathrm{CH}_{2}=\mathrm{CH}-\mathrm{CH}_{2}-\right), 5.09\left(\mathrm{~d}, 1 \mathrm{H}, J_{1,2} 1.2 \mathrm{~Hz}\right.$, $\left.\mathrm{H}-1^{\mathrm{II}}\right), 5.10\left(\mathrm{~d}, 1 \mathrm{H}, J_{1,2} 1.2 \mathrm{~Hz}, \mathrm{H}-1^{\mathrm{IV}}\right), 5.25$ (d, $\left.1 \mathrm{H}, J_{1,2} 3.6 \mathrm{~Hz}, \mathrm{H}-1^{\mathrm{III}}\right), 5.37-5.40(\mathrm{~m}, 2$ $\left.\mathrm{H}, \mathrm{H}-4, \mathrm{CH}_{2}=\mathrm{CH}-\mathrm{CH}_{2}-\right)$, 5.47-5.48 (m, $1 \mathrm{H}$, $\left.\mathrm{H}-2^{\mathrm{II}}\right), \quad 5.56-5.58 \quad\left(\mathrm{~m}, 2 \mathrm{H}, \mathrm{H}-3^{\mathrm{II}}\right.$, $\left.\mathrm{CH}_{2}=\mathrm{CH}-\mathrm{CH}_{2}-\right), 5.64\left(\mathrm{t}, 1 \mathrm{H}, J_{3,4} 10 \mathrm{~Hz}\right.$, $\left.\mathrm{H}-4^{\mathrm{II}}\right), 5.72-5.73\left(\mathrm{dd}, 1 \mathrm{H}, \mathrm{H}-2^{\mathrm{IV}}\right), 5.76-5.78$ (m, $\left.2 \mathrm{H}, \mathrm{H}-3^{\mathrm{IV}}, \mathrm{H}-4^{\mathrm{IV}}\right), 6.11-6.15\left(\mathrm{~m}, 2 \mathrm{H}, J_{3,4}\right.$ $\left.10 \mathrm{~Hz}, \mathrm{H}-3^{\mathrm{III}}, \mathrm{CH}_{2}=\mathrm{CH}-\mathrm{CH}_{2}-\right), 7.23-8.10(\mathrm{~m}$, $40 \mathrm{H}, 8 \mathrm{Ph})$. Anal. Calcd for $\mathrm{C}_{79} \mathrm{H}_{70} \mathrm{O}_{24}: \mathrm{C}$, 67.62; H, 4.99. Found: C, 67.47; H, 5.08.

Allyl 3,4,6-tri-O-benzoyl- $\alpha$-D-mannopyranosyl-( $1 \rightarrow 2)$ - [2,3,4-tri-O-benzoyl- $\alpha$-L-rhamnopyranosyl - $(1 \rightarrow 6)]-3,4-d i-\mathrm{O}-$ benzoyl $-\alpha-\mathrm{D}-$ glucopyranoside (12).-Compound 11 (196 $\mathrm{mg}, 0.14 \mathrm{mmol}$ ) was dissolved in $1: 1 \mathrm{CH}_{2} \mathrm{Cl}_{2}$ $\mathrm{CH}_{3} \mathrm{OH}(14 \mathrm{~mL}$ ), and $3 \%$ (volume ratio) of acetyl chloride was added. The reaction mixture was stirred at $\mathrm{rt}$ for $20 \mathrm{~h}$, at the end of which time, TLC (1:2 EtOAc-petroleum ether) indicated the reaction was complete. The solvent was neutralized with $\mathrm{Et}_{3} \mathrm{~N}$, concentrated and subjected to the column chromatography (2.5:1 petroleum ether-EtOAc) to give compound $12(152 \mathrm{mg}, 80 \%)$ as a syrup; $[\alpha]_{\mathrm{D}}^{25}+66^{\circ}\left(c \quad 0.9, \mathrm{CHCl}_{3}\right) ;{ }^{1} \mathrm{H} \mathrm{NMR}$ $\left(\mathrm{CDCl}_{3}\right): \delta 1.28\left(\mathrm{~d}, 3 \mathrm{H}, J 6.2 \mathrm{~Hz}, \mathrm{CH}_{3}\right), 3.76$ $\left(\mathrm{dd}, 1 \mathrm{H}, J_{5,6 \mathrm{a}} 7.2, J_{6 \mathrm{a}, 6 \mathrm{~b}} 12.0 \mathrm{~Hz}, \mathrm{H}-6 \mathrm{a}^{\mathrm{III}}\right), 3.92$ $\left(\mathrm{dd}, J_{5,6 \mathrm{~b}} 4.0, J_{6 \mathrm{a}, 6 \mathrm{~b}} 12.0 \mathrm{~Hz}, \mathrm{H}-6 \mathrm{~b}^{\mathrm{III}}\right), 4.11-$ 4.30 (m, $5 \mathrm{H}, \mathrm{H}-2^{\mathrm{III}}, \mathrm{H}-5^{\mathrm{IV}}, \mathrm{H}-5^{\mathrm{II}}, \mathrm{H}-6 \mathrm{a}^{\mathrm{II}}$, $\left.\mathrm{CH}_{2}=\mathrm{CH}-\mathrm{CH}_{2}-\right), 4.35-4.40\left(\mathrm{~m}, 2 \mathrm{H}, \mathrm{H}-5^{\mathrm{III}}\right.$, $\left.\mathrm{H}-2^{\mathrm{II}}\right), 4.46-4.51\left(\mathrm{~m}, 2 \mathrm{H}, \mathrm{CH}_{2}=\mathrm{CH}-\mathrm{CH}_{2}-\right.$, H-6b $\left.{ }^{\mathrm{II}}\right), 5.12\left(\mathrm{~d}, 1 \mathrm{H}, J_{1,2} 1.6 \mathrm{~Hz}, \mathrm{H}-1^{\mathrm{II}}\right), 5.14$ $\left(\mathrm{d}, 1 \mathrm{H}, J_{1,2} 1.6 \mathrm{~Hz}, \mathrm{H}-1^{\mathrm{IV}}\right), 5.29\left(\mathrm{~d}, 1 \mathrm{H}, J_{1.2}\right.$ $\left.3.6 \mathrm{~Hz}, \mathrm{H}-1^{\mathrm{III}}\right), 5.34-5.44$ (t, $3 \mathrm{H}, \mathrm{H}-4^{\mathrm{III}}, \mathrm{H}-3^{\mathrm{II}}$, $\left.\mathrm{CH}_{2}=\mathrm{CH}-\mathrm{CH}_{2}-\right)$, 5.52-5.55 (m, $1 \mathrm{H}$, $\left.\mathrm{CH}_{2}=\mathrm{CH}-\mathrm{CH}_{2}-\right), 5.66\left(\mathrm{t}, 1 \mathrm{H}, J_{3,4} 10 \mathrm{~Hz}\right.$, $\left.\mathrm{H}-4^{\mathrm{II}}\right), 5.72\left(\mathrm{dd}, 1 \mathrm{H}, \mathrm{H}-2^{\mathrm{IV}}\right), 5.76-5.79$ (m, 2 $\left.\mathrm{H}, \quad \mathrm{H}-3^{\mathrm{IV}}, \mathrm{H}-4^{\mathrm{IV}}\right), 6.00-6.09$ (m, $1 \mathrm{H}$, $\left.\mathrm{CH}_{2}=\mathrm{CH}-\mathrm{CH}_{2}-\right), 6.12\left(\mathrm{t}, 1 \mathrm{H}, J_{3,4} 10 \mathrm{~Hz}\right.$, $\left.\mathrm{H}-3^{\mathrm{III}}\right)$, 7.25-8.09 (m, $\left.40 \mathrm{H}, 8 \mathrm{Ph}\right)$. Anal. Calcd for $\mathrm{C}_{77} \mathrm{H}_{68} \mathrm{O}_{23}$ : C, 67.94; H, 5.00. Found: C, 67.68; H, 5.16.

Allyl 2,3,5-tri-O-benzoyl- $\alpha$-D-arabinofuranosyl-( $1 \rightarrow 2)$ - 3,4,6-tri-O-benzoyl- $\alpha$-D-mannopyranosyl - $(1 \rightarrow 2)-[2,3,4$ - tri - O - benzoyl - $\alpha$ - L- 
rhamnopyranosyl-(1 $\rightarrow 6)]-3,4-$ di-O-benzoyl- $\alpha-$ D-glucopyranoside (14). - To a mixture of compound 12 (100 $\mathrm{mg}, 0.07 \mathrm{mmol})$ and $13^{18}$ (48 $\mathrm{mg}, 0.08 \mathrm{mmol})$ in anhyd $\mathrm{CH}_{2} \mathrm{Cl}_{2}(5 \mathrm{~mL})$ was added $\mathrm{Me}_{3} \mathrm{SiOTf}$ (15\% equiv) under a $\mathrm{N}_{2}$ atmosphere at $0{ }^{\circ} \mathrm{C}$. The mixture was stirred under these conditions for $3 \mathrm{~h}$, then neutralized with $\mathrm{Et}_{3} \mathrm{~N}$ and concentrated. The residue was purified by column chromatography (2:1 petroleum ether-EtOAc) to give $14(106 \mathrm{mg}$, $80 \%)$ as a syrup; $[\alpha]_{\mathrm{D}}^{25}+65^{\circ}\left(c 0.7, \mathrm{CHCl}_{3}\right)$; ${ }^{1} \mathrm{H}$ NMR $\left(\mathrm{CDCl}_{3}\right): \delta 1.28(\mathrm{~d}, 3 \mathrm{H}, J 6.4 \mathrm{~Hz}$, $\mathrm{CH}_{3}$ ), $3.72\left(\mathrm{dd}, 1 \mathrm{H}, J 7.0,12.0 \mathrm{~Hz}, \mathrm{H}-6 \mathrm{a}^{\mathrm{III}}\right)$, $3.90\left(\mathrm{dd}, 1 \mathrm{H}, J 2.3,12.0 \mathrm{~Hz}, \mathrm{H}-6 \mathrm{~b}^{\mathrm{III}}\right), 4.11-$ $4.26\left(\mathrm{~m}, 6 \mathrm{H}, \mathrm{H}-2^{\mathrm{III}}, \mathrm{H}-5^{\mathrm{IV}}, \mathrm{H}-5^{\mathrm{II}}, \mathrm{H}-6 \mathrm{a}^{\mathrm{II}}\right.$, $\left.\mathrm{H}-6 b^{\mathrm{II}}, \mathrm{CH}_{2}=\mathrm{CH}-\mathrm{CH}_{2}-\right)$, 4.35-4.42 (m, $2 \mathrm{H}$, $\left.\mathrm{H}-5^{\mathrm{III}}, \quad \mathrm{H}-2^{\mathrm{II}}\right), \quad 4.44-4.46 \quad(\mathrm{~m}, 1 \mathrm{H}$, $\left.\mathrm{CH}_{2}=\mathrm{CH}-\mathrm{CH}_{2}-\right), 4.61-4.63(\mathrm{~m}, 2 \mathrm{H}, \mathrm{H}-4$, H-5 $\left.\mathrm{a}^{\mathrm{I}}\right), 4.69\left(\mathrm{dd}, 1 \mathrm{H}, J\right.$ 5.0, $\left.11.4 \mathrm{~Hz}, \mathrm{H}-5 \mathrm{~b}^{\mathrm{I}}\right)$, $5.10\left(\mathrm{~d}, 1 \mathrm{H}, J_{1,2} 1.2 \mathrm{~Hz}, \mathrm{H}-1^{\mathrm{II}}\right), 5.21(\mathrm{~d}, 1 \mathrm{H}$, $\left.J_{1,2} 3.6 \mathrm{~Hz}, \mathrm{H}-1^{\mathrm{III}}\right), 5.24\left(\mathrm{~d}, 1 \mathrm{H}, J_{1,2} 1.2 \mathrm{~Hz}\right.$, $\left.\mathrm{H}^{-1}{ }^{\mathrm{IV}}\right), 5.29\left(\mathrm{~d}, 1 \mathrm{H}, J 10.4 \mathrm{~Hz}, \mathrm{H}-3^{\mathrm{II}}\right), 5.37$ (t, $\left.1 \mathrm{H}, \mathrm{H}-4^{\mathrm{III}}\right), 5.45-5.49(\mathrm{dd}, 1 \mathrm{H}, J 1.2 \mathrm{~Hz}$, $\left.\mathrm{CH}_{2}=\mathrm{CH}-\mathrm{CH}_{2}-\right), 5.47\left(\mathrm{~s}, 1 \mathrm{H}, \mathrm{H}-3^{\mathrm{I}} / 2^{\mathrm{I}}\right), 5.60-$ $5.67\left(\mathrm{~m}, 4 \mathrm{H}, \mathrm{H}-1^{\mathrm{I}}, \mathrm{H}-4^{\mathrm{II}}, \mathrm{CH}_{2}=\mathrm{CH}-\mathrm{CH}_{2}-\right.$, $\left.\mathrm{H}-2^{\mathrm{I}} / 3^{\mathrm{I}}\right), 5.71\left(\mathrm{dd}, 1 \mathrm{H}, \mathrm{H}-2^{\mathrm{IV}}\right), 5.78(\mathrm{dd}, 1 \mathrm{H}$, $\left.J_{2,3} 3.2, J_{3,4} 12 \mathrm{~Hz}, \mathrm{H}-3^{\mathrm{IV}}\right), 5.84\left(\mathrm{t}, 1 \mathrm{H}, J_{3,4} 12\right.$ $\left.\mathrm{Hz}, \quad \mathrm{H}-4^{\mathrm{IV}}\right), \quad 5.96-6.04 \quad(\mathrm{~m}, \quad 1 \mathrm{H}$, $\left.\mathrm{CH}_{2}=\mathrm{CH}-\mathrm{CH}_{2}-\right), 6.12\left(\mathrm{t}, 1 \mathrm{H}, J_{3,4} 10 \mathrm{~Hz}\right.$, H-3 $\left.{ }^{\mathrm{III}}\right), 7.23-8.10(\mathrm{~m}, 55 \mathrm{H}, 11 \mathrm{Ph})$, MALDITOF-MS Calcd for $\mathrm{C}_{103} \mathrm{H}_{88} \mathrm{O}_{30} \mathrm{Na}^{+}$1827.5; Found $m / z 1827.3\left(\mathrm{M}+\mathrm{Na}^{+}\right)$. Anal. Calcd for $\mathrm{C}_{103} \mathrm{H}_{88} \mathrm{O}_{30}: \mathrm{C}, 68.51 ; \mathrm{H}, 4.88$. Found: $\mathrm{C}$, 68.77; H, 4.74.

\section{Acknowledgements}

This work was supported by CAS KIPRCEES9904, KJ952 J 561, and NNSF of China (Projects 29972053 and 39970179).

\section{References}

1. Groves, P.; Searle, M. S.; Waltho, J. P.; Williams, D. H. J. Am. Chem. Soc. 1995, 117, 7958-7964.

2. Xu, R.; Greiveldinger, G.; Marenus, L. E.; Cooper, A.; Ellman, J. A. J. Am. Chem. Soc. 1999, 121, 4898-4999.

3. Sztaricskai, F.; Lipták, A.; Pelyvás, I. F.; Bogánr, R. J. Antibiot. 1976, 24, 626-631.

4. Neszmélyi, A.; Sztaricskai, F.; Lipták, A.; Bognár, R. J. Antibiot. 1978, 26, 974-978.

5. Williamson, M. P.; Williams, D. H. Tetrahedron Lett. 1980, 21, 4187-4188.

6. Sztaricskai, F.; Harris, C. M.; Neszmélyi, A.; Harris, T. M. J. Am. Chem. Soc. 1980, 102, 7093-7099.

7. Sztaricskai, F.; Neszmélyi, A.; Bognár, R. Tetrahedron Lett. 1980, 21, 2983-2986.

8. Barna, J. C. J.; Williams, D. H.; Williamson, M. P. J. Chem. Soc., Chem. Commun. 1985, 254-256.

9. Du, Y.; Pan, Q.; Kong, F. Synlett 1999, 1648-1650.

10. Du, Y.; Zhang, M.; Kong, F. Chinese Pat. Appl. 00107723.6 (2000).

11. Ding, X.; Wang, W.; Kong, F. Carbohydr. Res. 1997, 303, $445-448$.

12. Lowary, T. L.; Eichler, E.; Bundle, D. R. J. Org. Chem. 1995, 60, 7316-7327.

13. Du, Y.; Pan, Q.; Kong, F. Carbohydr. Res. 2000, 323, 28-35.

14. Pearson, A. J.; Heo, J.-N. Tetrahedron Lett. 2000, 41, 5991-5996.

15. Whistler, R. L.; Wolfrom, M. L. Methods Carbohydr. Chem. 1963, 2, 326-327.

16. Mulard, L. A.; Ughetto-Monfrin, J. J. Carbohydr. Chem. 1999, 18, 721-753.

17. Ogawa, T.; Katano, K.; Sasajima, K.; Matsui, M. Tetrahedron 1981, 37, 2779-2786.

18. Du, Y.; Pan, Q.; Kong, F. Carbohydr. Res. 2000, 329, $17-24$. 